\title{
Non-surgical Management of Pierre Robin Syndrome
}

\author{
W. D. PIELOU \\ From the School of Dentistry, Queen's University of Belfast
}

\begin{abstract}
A review of relevant published reports seems to indicate that in the case of babies suffering from intermittent airway obstruction due to a combination of micrognathia, glossoptosis, and often cleft of hard and soft palate (the Pierre Robin syndrome), successful treatment usually includes surgery of one kind or another. However, some non-surgical methods have been advocated. Pierre Robin (1934) recommended 'orthostatic' feeding, where the baby is forced to suck at the mother's breast with the thorax held up straight, to lift the neck and propel the chin forward. Davis and Dunn (1933) devised an attachment to the feeding bottle of a curved plate which rested on the upper lip and forced the baby to extend the lower jaw. Eley and Farber (1930) used a head cap supporting a strong copper wire which passed down behind the ramus on each side and under the chin. This wire was adjusted so as to keep the mandible in a forward position.
\end{abstract}

Dennison (1965), in his paper reviewing 44 case histories, states that a cleft palate is a common but not constant feature of the syndrome. He lists the different types of management required for his patients and suggests that surgery has little to offer in the treatment of the syndrome.

Of the surgical treatments, the Douglas (1946) technique, whereby the mucous membrane was removed from the anterior tongue and inner margin of the lower lip and these parts then sutured together, appears to work well providing the surgical union heals without complications.

Other surgical methods included wires through and round the anterior mandible to hold it forward by cords or springs attached to external framework or weights.

Hadley and Johnson (1963) described a successfully treated case using a Kirschner wire which was passed in through one ramus, and out through the other. The wire was inserted low enough so as to pass through the posterior body of the tongue, thus fixing it in the forward position.

With regard to tracheostomies, Moyson (1961) calls this type of treatment for the Pierre Robin

Received May 11, 1966. syndrome baby in severe respiratory distress 'a solution of defeat', and he makes a plea against tracheostomy, describing how complications tend to overwhelm the self-defence of the patient.

Before the non-surgical treatment described in this paper was started, babies born in this area with the Pierre Robin syndrome and in danger of asphyxiation were referred for tracheostomy. In a five-year period 13 such tracheostomies were performed, in each instance the tube in the trachea was in situ until repair of the palatal defect was carried out one year later; 12 of these were satisfactory with no complications due to the tracheostomy. The remaining case had a successful tracheostomy but died at 6 months due to a large cardiac defect.

In cases where surgical methods have been used gastric tube and spoon methods of feeding are the rule, so that the tongue gets very little active work to do, and in consequence there is little chance of improving its muscular tone.

Apart from those babies suffering from respiratory difficulty at birth and after, Routledge (1960) felt that there was the possibility of a respiratory crisis occurring several weeks later in an initially unobstructed baby as the result of the relatively faster rate of growth of the tongue as compared with the mandible.

Goldberg and Eckblom (1962) are the only authors to mention the use of an obturator to allow nipple feeding, and then only after the surgical part of the treatment consisting of drawing the tongue forward by external traction was completed.

\section{Treatment}

The treatment described here is an attempt to maintain an adequate airway in these babies without having to resort to operation. Babies now presenting with the Pierre Robin syndrome, whether in respiratory distress or not, are seen and fitted with a special obturator. In a two-year period 11 cases have been seen and 10 treated in this manner. Of the 10 cases, 3 had frequent attacks of cyanosis relieved by placing on the face, and exaggerated by 


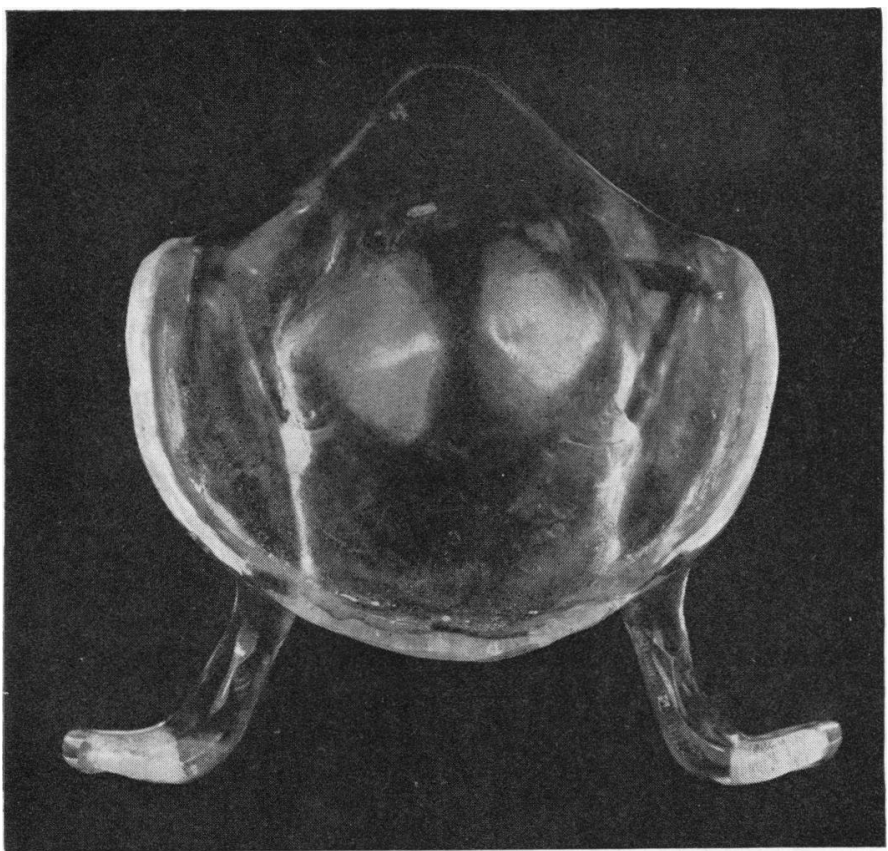

FIG. 1.-The obturator with extra oral 'wings' and distal extension to the palate. It is made of clear acrylic.

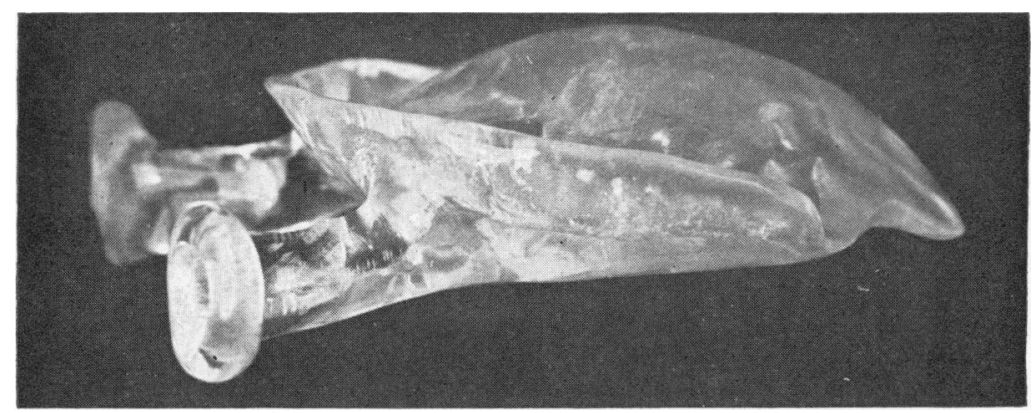

FIG. 2.-Lateral view of the obturator showing the curve of the disto-palatal extension.

placing on the back. Similar attacks were produced on feeding and frequently all or part of a feed would be vomited immediately after being given. The other $7 \mathrm{had}$ feeding problems only.

The eleventh child, though having all the signs but not the symptoms, of a severe Pierre Robin syndrome case, reacted violently to an obturator of any kind in his mouth and his mother stated that she had no feeding or other problems relevant to the child's oral condition.

The patients are seen as soon as possible after birth, when an impression is taken of the upper jaw, using a white impression compound of low soften- ing temperature. From this impression an obturator is made in clear acrylic plastic and inserted in the baby's mouth, usually within four hours of the impression being taken.

As illustrated in Fig. 1 and 2, the obturator has an extension posteriorly beyond the distal korder of the palate and extensions, or wings, anteriorly, which are useful on insertion and removal, though their main function is to allow the plate to be taped, the tapes being tied behind the baby's head. These tapes are prevented from slipping by sticking over them on the cheeks small pieces of plastic sticky tape specially made so as not to cause inflammation of the skin. 


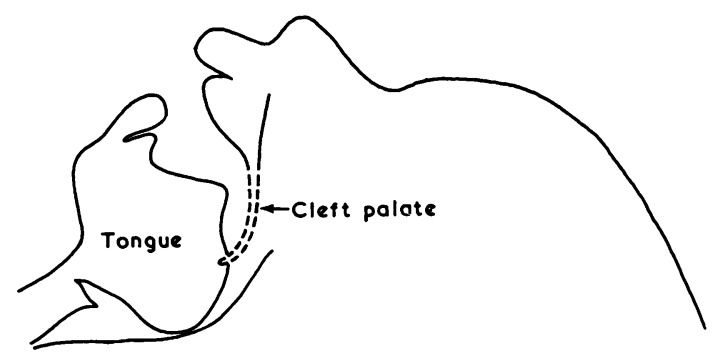

FIG. 3.-Outline drawing showing the position of the tongue.

The primary object of the obturator is to prevent the tongue from falling back into the pharynx, and in order to do this it must have an extension curving distally and downwards from the distal border of the obturator.

The outline drawings in Fig. 3 and 4 indicate the tongue position before and after insertion of the obturator. To place such an obturator in the mouth of an older child or an adult would produce immediate retching and gagging as soon as the extension came in contact with the sensitive posterior part of the tongue. Fortunately in the newborn this sensitivity is much less, but should reaction continue then some removal of the distal extension is indicated.

It has been noted that usually the baby who retches and gags most easily when a foreign body is placed at the back of the mouth has also a more active tongue and appears to be able to retain it in the forward position without aid. In this case, the obturator is of use as an aid to bottle feeding only by closing the hard palate defect. Hence the distal extension is unnecessary and may be removed entirely.

It is interesting to note that the placing of the obturator in the mouth not only helps to prevent the tongue falling back, but also encourages the baby to use its tongue more than it would otherwise do, and sucking on the obturator begins almost at once. The period of adjustment to an obturator by an adult is usually hours or even days, but in the case of these babies it is a matter of minutes, so that soon the baby objects strongly to having it removed for cleaning after feeds, etc.

As is well known, if these children can be kept alive and thriving then the rapid growth of the mandible will eventually eliminate the adverse symptoms of the syndrome within a few months. It is felt that an aspect just as important in this respect is the improvement of the neuromuscular condition of the tongue and the establishment of the

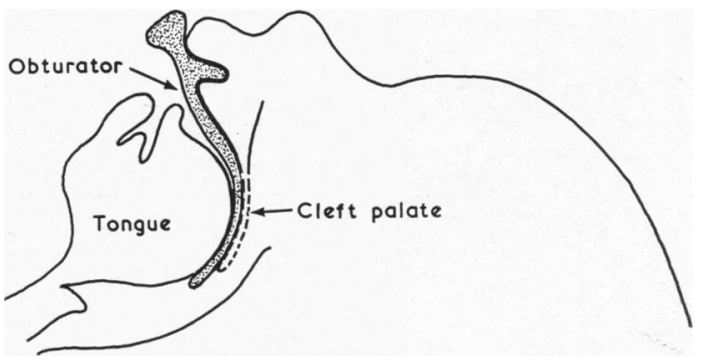

FIG. 4.-Outline drawing showing the position of the tongue after insertion of the obturator.

habit of keeping the tongue forward in the mouth.

Such a state of affairs is brought about much more rapidly if the baby is made to suck actively rather than be fed by dropper or spoon.

\section{Illustrative Case History}

The baby was brought to the Royal Belfast Hospital for Sick Children 12 days after birth. The birthweight was $3528 \mathrm{~g}$. and delivery was normal. Since birth she had frequent cyanotic attacks, especially following feeding, and her breathing was always noisy. She had been spoon-fed from birth as she was unable to suck, and she never finished a feed without vomiting. The food frequently came down her nose.

An obturator as described above was inserted, following which no further episodes of cyanosis occurred. Sucking was at first very slow, but this rapidly improved and two weeks later the sucking reflex was noted as 'very strong' and gain in weight was satisfactory.

The lateral view (Fig. 5) taken two weeks after insertion of the obturator (not fitted here) shows the typical retroposition of the chin in the Pierre Robin syndrome. The marks on the cheek are due to the use of zinc oxide tape to prevent the head tapes from slipping up or down. A special plastic tape which is innocuous to tender skin and which is commercially available is now used.*

One month later a new plate was fitted which continued to be very satisfactory until at 6 months the baby refused to keep it in her mouth, at which time it was discarded without ill effect.

In Fig. 6 the tapes shown are not supporting the obturator firmly in its correct position, but are merely preventing it from being dislodged. In spite of the fact that the baby is crying, the obturator is still seated well up in the palate so that only the extra-oral wings are visible. This is usual with an accurately fitting obturator.

\section{Summary}

Following a brief review of the published reports on the treatment of the Pierre Robin syndrome, a

^ Band aid (Trademark) 'clear Tape' (Johnson and Johnson, U.S.A.). 


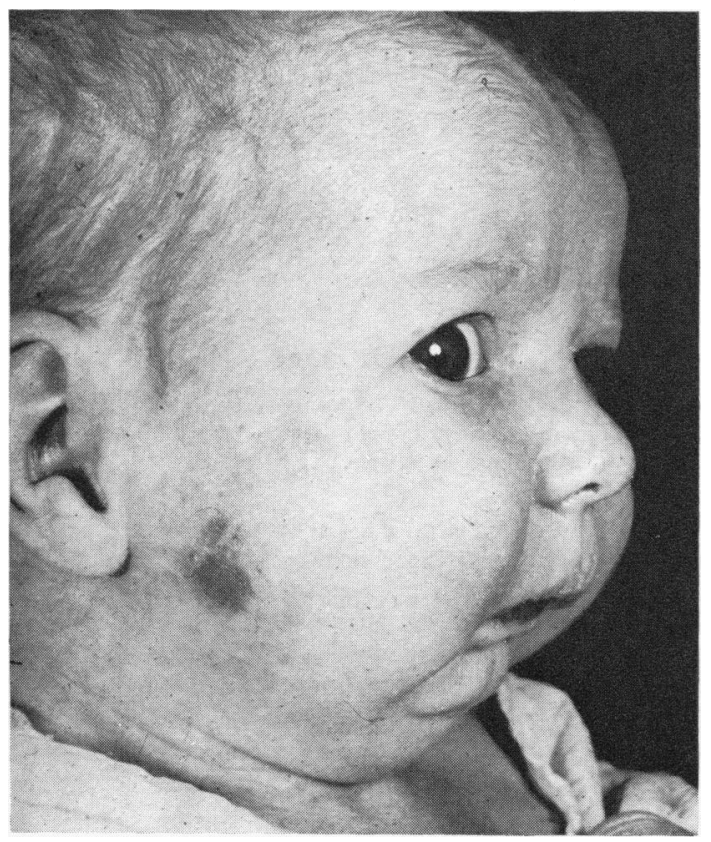

FIG. 5.-Age 2 weeks: the retro-position of the chin is well seen here.

non-surgical management is described, making use of an acrylic obturator similar to a dental plate, but having a distal extension which prevents the tongue falling back into the pharynx.

Ten cases have been treated.

\section{REFERENCES}

Davis, A. D., and Dunn, R. (1933). Micrognathia. A suggested treatment for correction in early infancy. Amer. F. Dis. Child., 45, 799.

Dennison, W. M. (1965). The Pierre Robin syndrome. Pediatrics, 36, 336.

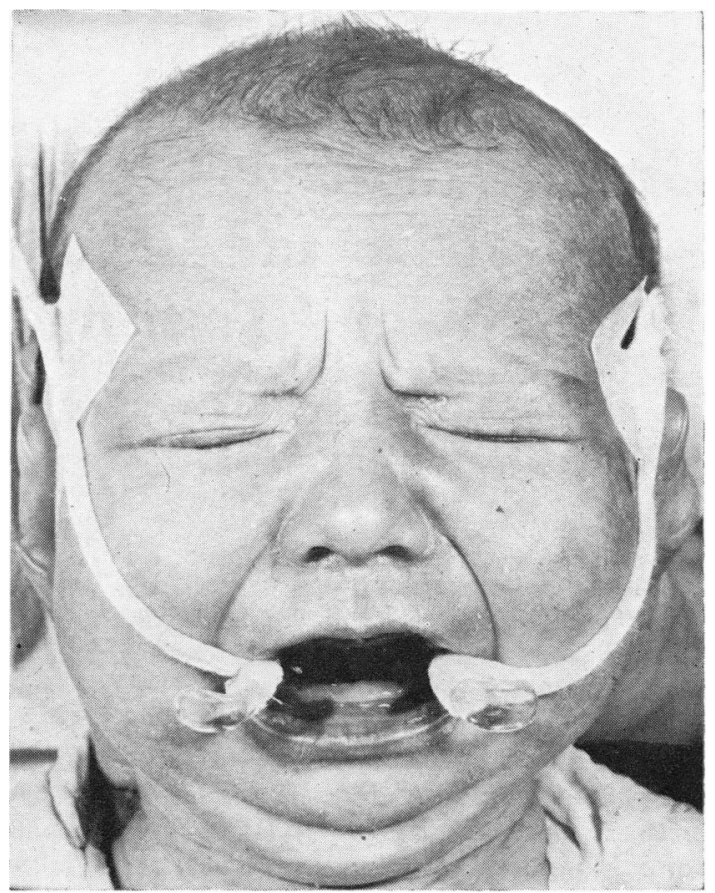

FIG. 6.-Age 2 weeks. Front view of baby with the obturator in position (note: though the baby is crying the obturator has remained well up in the palatal area).

Douglas, B. (1946). The treatment of micrognathia associated with obstruction by plastic procedures. Plast. reconstr. Surg., 1,300 . Eley, R. C., and Farber, S. (1930). Hypoplasia of the mandible. Amer. F. Dis. Child., 39, 1167.

Goldberg, M. H., and Eckblom, R. H. (1962). The treatment of the Pierre Robin syndrome. Pediatrics, 30, 450.

Hadley, R. C., and Johnson, J. B. (1963). Utilization of the Kirschner wire in Pierre Robin syndrome with case report. Plast. reconstr. Surg., 31, 587.

Moyson, F. (1961). A plea against tracheostomy in the PierreRobin syndrome. Brit. F. plast. Surg., 14, 187.

Robin, P. (1934). Glossoptosis due to atresia and hypotrophy of the mandible. Amer. F. Dis. Child., 48, 541.

Routledge, R. T. (1960). The Pierre-Robin syndrome: a surgical emergency in the neonatal period. Brit. F. plast. Surg., 13, 204. 